\title{
Augmenting Instructional Design with State-Based Assessment
}

\author{
Kevin Oden \\ Lockheed Martin Corporation, Orlando Florida, USA \\ Kevin.oden@lmco.com
}

\begin{abstract}
The Trainee Engagement Management System (TEMS) is a technology-enabled instructional design concept that leverages state-based assessment techniques to improve training processes and outcomes. Specifically, the concept is designed to support military instructors in the delivery of empirically-supported instructional prompts to foster trainee engagement within a Computer Based Training (CBT) environment. The central theme of the concept is to augment, not replace, an instructor's abilities. By reducing workload demands on an instructor, the approach enables the delivery of personalized instruction in a one (instructor) to many (trainees) context. The TEMS concept embraces a human-system philosophy and is designed to mitigate risks typically associated with the transition of advanced technologies and concepts to field settings. In this paper we discuss those challenges and describe the basic TEMS architecture.
\end{abstract}

Keywords: Instructional System Design, Augmented Cognition, Human Systems, Computer Based Training.

\section{Introduction}

Under conditions of persistent conflict and mounting economic pressures military instructors are required to impart mission-critical Knowledge, Skills, Abilities (KSAs) with fewer resources. Training remains as the primary mechanism for acquiring and maintaining operational readiness across all branches of the military. Many opportunities exist within instructional design field(s) to support the efficient and affordable delivery of high quality instruction. However, a co-occurrence of change across science, technology, and Operational Environments (OEs) often disrupts the successful transition of innovative and validated instructional designs to field settings. In part, a lack of coordination across these fields contributes to a growing tension as to how 'advanced' should be defined with respect to instructional technology. Researchers from each field, in earnest, work to accomplish a shared goal - improve Operator performance; however, they tend to pursue orthogonal objectives that rarely converge to produce a field-ready solution.

In this paper, we describe a technology enabled instructional design concept that embeds monitoring and management strategies to facilitate trainee engagement in 
Computer Based Training (CBT) settings. The primary objective is to sharpen and sustain focused attention of trainees in the service of learning. A brief review of relevant literature and contemporary work is provided to illustrate how advanced physiological technology and learning concepts can be combined to augment instructor performance and promote training effectiveness and efficiency.

\section{Human-System Components}

A need for field-ready instructional technologies that incorporate best offerings from the aforementioned fields has been elevated. Cross-Cutting instructional designs that leverage sufficiently mature technologies and validated learning practices are the best candidates for transition to field settings. It is clear that training system success is build upon many human factors, but it is not obvious how human should be integrated as components within the total system. An instructional design concept that integrates/balances the following instructional system components is most likely to achieve success that can be transitioned to the field in the near-term: trainee, instructor, and instructional technology.

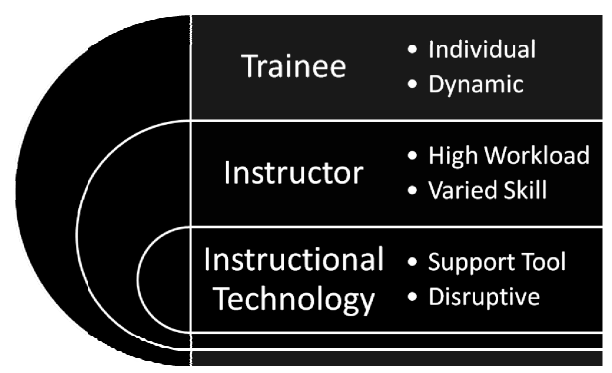

Fig. 1. Targeting Fundamental Elements of Instructional System

The components presented above (see, Figure 1) represent the essential features common across training systems. From a human-systems perspective, each component piece must be carefully considered as trade-offs are made during design. The interplay between the components is ultimately what determines total system success. Specifically, the goodness of training systems is directly proportional to a directional change in measures of trainee performance. Typically, a training system will be evaluated based on metrics of effectiveness (i.e., trainee obtains a new KSA) and efficiency (i.e., how long it took to obtain a given KSA).

Individual differences across a trainee population impacts training processes and outcomes related to effectiveness and efficiency. Each new training event is meaningfully affected by both inter-personal and intra-personal factors. For instance, a trainee's full history of learning experiences is an inter-personal factor that uniquely defines their KSA baseline. Equivalent amounts time spent on the same task may increase similarity between two trainees, but individual differences will persist. 
Additionally, human variations within a trainee may color a training event, so that outcomes on identical tasks are not consistent for a given trainee over time. These dynamic intra-personal factors may change from moment-to-moment and are often classified as state-based variables. For example, a person's ability to focus on a task rarely remains stable over extended periods of time, instead fluctuations from highly engaged to extreme bored are normal. The mix of inter-personal and intra-personal differences prohibits a 'one size fits all' approach to training system design. Therefore, instructional designs must include adaptable features that afford individualized tailoring to maximize each training events for each trainee.

The second component, the instructor, is the primary resource currently available for providing personalized tailored training. In a one-to-one context an instructor can reasonably estimate a trainee's state and make real-time adjustments to better match instruction to a trainee's current needs in real-time within a training context. Unfortunately, the one-to-one approach is not cost a cost effective method, nor is it reasonable from a manpower perspective; skilled personnel/instructors are a valuable resource. A more common approach is to have a single instructor provide CBT to multiple trainees at a time, thus, diminishing the value of the highly skilled instructor. Monitoring and managing multiple trainees overwhelms an instructor's mental bandwidth making it nearly impossible to estimate a trainee's current needs and make instructionally significant adjustments. Moreover, the high workload requirements placed on instructors becomes more troubling for novice instructors that lack the abilities gained through experience.

The final component, the instructional technology, is the mediating component that has the greatest potential to enhance, or disrupt, the training environment. In a CBT context, the technology (hardware and software) connects both the trainee and instructor to the instructional materials and it connects them to each other. Thus, favorable outcomes are directly related to the quality of the human system integration. Unfortunately, this important point is often overlooked because the objectives of a technologist are derived from goals that are indirectly linked to the basic premise of instruction, impart knowledge and skills. The disconnect results in an increased risk for the development of ineffective instructional tools. For example, improvements in 3D graphics may improve collaboration work, but this is of little value to an instructor that is training KSAs for a one-person task. Moreover, instructional technologies with form factors that don't match the training environment are not good candidates for transition to field settings. Therefore, a human-systems integration philosophy is particularly useful for the design, development, and implementation of advanced instructional concepts and technology.

\subsection{Human Performance}

Over the past one hundred years, models of human performance and behavior have indicated that KSAs tend to fit within scoped boundaries, such as, the inverted Uhypothesis [1], zone of proximal development [2], comfort zone [3], and flow state [4]. The overarching take-away from the extant literature is that there appears to be a "sweet spot" for getting people to perform at their best! While it appears that we all 
have a sweet spot it is also clear that finding the sweet spot is highly individual. What stimulates the flow state in one person may frustrate, or bore, someone else. Moreover, it is not easy to get into or maintain this highly desirable state of optimal performance. There are techniques that can help initiate flow; however, they require meta-cognitive skills, such as, self monitoring and regulation of cognitive states. Unfortunately, timely attainment of these higher order skills is likely beyond the reach of most people. These are implicit skills that are difficult to quantify and are not readily observable. However, the field of augmented cognition is making strides toward the design of technology/tools that may make these implicit processes explicit. In the near-term, these technologies may provide insight to instructors about a trainee's state that may enable for the design of systematic methods to stimulate a flow state. An instructional design that integrates the human-technology components so tightly has great potential to deliver personalized instruction in a number of new and interesting ways.

\section{* But lo! Men have become the tools of their tools, Thoreau, Henry David}

In 1854, Henry David Thoreau raised an idea (maybe concern) to emphasize the inter-relation between people and technology. Leastwise, his statement illustrates how technology "tools" are more than mere objects; they are integral implements that interact with people to facilitate achievement of goals. Often, advanced technologies are not accepted by military leaders and/or instructors because they are too disruptive, either in concept of operations or technical execution. But, also, there is this idea of the status quo in which people are comfortable using current tools in the manner to which they have grown accustom. Whether, or not, the new tool is better suited for the task at hand is moot if it is judged to be too farfetched. To balance the acceptanceadvanced equation, the initial Trainee Engagement Management System (TEMS) solution is being designed as an enhancement to a government owned Instructor Operator Station (IOS) that aligns with practices currently employed by military instructors. In short, introducing a simple design modification to an existing CBT context might be an acceptable application of advanced physiological technologies.

\subsection{Instructional Quality}

In broad terms, instructional quality can be tailored through monitoring and management of two types of variables - situation and person. Situation variables are the external factors and conditions of an instructional context that frames learning events. Because CBT platforms afford relatively easy monitoring and management of situation variables, it continues to be a very popular and useful option for the delivery of many instructional methods, such as, demonstration based training and simulation based training. Conversely, there remains a weakness in the instructional administration of CBT for the direct observation and management of person variables, such as cognition, affect, and attention that influence instructional quality. Maintaining engagement, or focused attention, is a very important person variable for knowledge and skill acquisition. Finding ways to stay focused is challenging for everyone. Our lives are filled with many things that compete for our attention, creating distraction and 
depleting our mental resources. In the case of military personnel that are immersed in a culture of always on technology it is common for them to lack the mental energy required to maintain focused attention during CBT. If a trainee is not engaged in the CBT event the path to learning is blocked impairing both training effectiveness and efficiency. The approach described in this paper acknowledges that trainee engagement is a cornerstone to the development of high performers. Specifically, comprehension and retention are affected by the trainee's degree of active participation (i.e., effort) in the training event. Paas [5] describes how the path to expertise depends on an individual's willingness to work at becoming expert:

* In research on deliberate practice, it has been noted that because this type of practice requires trainees to stretch themselves to a higher level of performance, it requires full concentration and is effortful to maintain. This does not make it a very enjoyable experience, so without the motivation to improve, trainees will soon give up (Ericsson et al., 1993). Feedback can play a crucial role in their willingness to continue to invest effort (indeed, feedback is also considered to play a crucial role in deliberate practice; see, e.g., Ericsson \& Lehmann, 1996). [6]

Ideally, each trainee would be drive by an intrinsic motivation to achieve expert status and, to that end, would supply the effort required to maintain engagement during a given training event. However, that ideal circumstance is not typical of trainee populations in real world contexts, nor is it reasonable to expect that any individual could consistently sustain that level of motivation. Thus, instructional designs that systematically foster active learning in typical training environments are desirable.

\section{Instructional Design Concept}

The TEMS design concept offers an augmented cognition solution to close the person variable loop. Augmented cognition is a field of research that continues to re-design Human Computer Interaction (HCI), as it makes technological systems responsive to state-based person variables. An opportunity exists to exploit Commercial Off-TheShelf (COTS) technologies that assess physiological arousal via measures of Electrodermal Activity (EDA) to improve instructional effectiveness and efficiency of CBT approaches. A new class of EDA technologies has successfully transitioned from controlled laboratories to real world settings. With improved form factors and low cost, these technologies are good candidates for near-term advancements in training contexts. Reliably, EDA measures arousal and can provide indications of high arousal (e.g., excitement, engagement, anger) and low arousal (e.g., boredom, disengagement, calm) that affect cognition and emotion. We have conceptualized an instructional design concept that utilizes EDA to mitigate information loss in a CBT context

Trainee engagement is a critical component for optimizing instructional effectiveness and efficiency of CBT. Often, a single instructor simultaneously administers CBT exercises to multiple trainees making it difficult for them to detect every 
occurrence of poor engagement and/or motivation. Thus, they are unable to provide crucial feedback or prompts that would re-engage the trainee. A key capability of TEMS is reporting to instructors those trainees lacking proper engagement during training exercises [6]. The TEMS concept focuses on the management of trainee engagement to maximize learning opportunities. To accomplish that we conceptualized a design that enhances an instructor's awareness of trainees' state to augment their judgments and improve decision making.

At one location, an instructor is able to monitor both behavioral performance and physiological indicators of a trainee's arousal. These two pieces of information combine to convey a more complete picture of a trainee's performance, or progress. In many instructor operator stations (IOSs) the instructor has the option to peer into a trainee's lesson so they can observer trainee performance. However, the ability to peer into a trainee's lesson only provides observations of situational variables - a very limited assessment of performance as discussed earlier in this paper. Paring a physiological assessment of arousal with the standard capability to observe trainee behaviors will augment instructor's ability to provide instructional support.

From a human-system perspective, the core capabilities of the TEMS instructional design concept are embodied in the IOS. The IOS contains the key systems features that enable the advanced training capabilities of the TEMS design concept. Moreover, the IOS is the system interface to the intelligence that drives the technology, the instructor. At the IOS, an instructor is able to monitor trainees' behaviors (performance) and physiological states (processes). The enhanced monitoring feature within the IOS enables the deployment of re-engagement strategies promoting a transactional model for instructional design, see Figure 2 below.

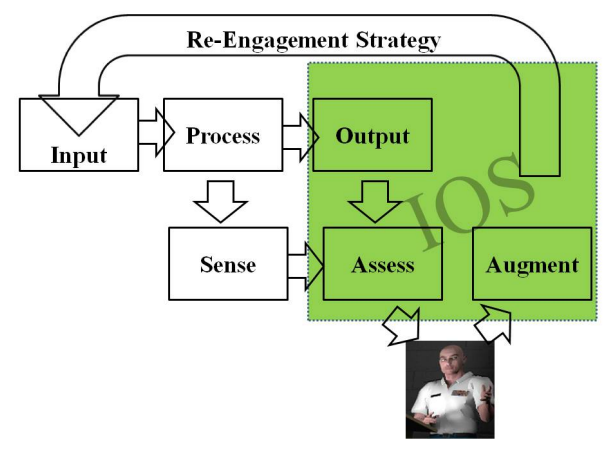

Fig. 2. Transactional Model for Instructional Technology

The transactional model for instructional design incorporates two process models: 1) Information-Process Output (IPO) for human performance, and 2) sense-assessaugment for human performance augmentation. The top three IPO boxes, in Figure 2, are conceptualized as follows; all stimuli in the world are symbolized as 'input', cognitive and biological processes are 'process', and behavioral actions taken are represented as 'outcome'. The United States Air Force's Human Performance Augmentation model is represented in the second row of 3 boxes and is conceptualized as; 
sensor monitoring provides 'sense', bio-data classification acts as 'assess', and intervention strategies assume the role of 'augment'[7]. As a foundation for TEMS, these models are used to characterize trainee performance so that the interplay between trainee behavioral performance and physiological arousal are exposed to instructors to enhance their awareness via an IOS.

Note, the instructor is in the loop and is responsible for deploying re-engagement content to the trainees. The prototype system will include an empirically supported metacognitive prompting strategy as its re-engagement strategy. Many opportunities exist to be creative with respect to designing re-engagement strategies and much research is needed to clearly link strategies to specific types of trainee needs. However, recent work suggests that the State-based Information-loss Process (SIP) Model could be used to appropriately administer a metacognitive prompt strategy.

\subsection{Advanced Learning Concepts}

The SIP Model was used to inform the initial prototype of the TEMS design concept. The SIP Model [8] identifies possible points for cognitive breakdown that may contribute to information loss during instruction. It is an evidence-supported model that focuses on higher-order learning (i.e., knowledge/skill integration and application) that is important for imparting complex KSAs that are required in military operations. Based on the SIP Model, we incorporated a metacognitive prompting strategy that could be used to mitigate information loss in a CBT context.

\section{* Learning without thought is labor lost, Confucius}

Metacognition is widely accepted as an implicit "thinking" skill that enhances one's ability to learn and solve problems [9]. Recent findings from the Nextgeneration Expeditionary Warfare-Intelligent Training (NEW-IT) program have demonstrated increases in learning effectiveness when metacognitive prompts were employed in the service of learning [10]. Those successes demonstrate that these prompts can be effective in a CBT context. We will build on those findings to develop a system that will help instructors more precisely target trainees for instructional intervention, metacognitive prompts. Leveraging a COTS state-based assessment of trainee arousal (i.e., EDA) the TEMS concept re-designs how this validated instructional strategy could be implemented. The physiological-based aspect of TEMS provides an objective assessment a person variable that allows instructors to more effectively, efficiently, and confidently employ an advanced strategy with a familiar CBT context. In addition to a meta-cognitive prompting strategy that we plan to test in the initial instantiation of the TEMS design concept, other prompting strategies could easily be adapted. Paas [5] outlines a few candidate prompting strategies that may be employed to promote deep comprehension and self regulation:

- Reflection prompts: to promote self-regulated learning competency and sustainability through reflection on one's own learning

- Self-explanation prompts: to promote understanding of the underlying principles of a problem, often provided with worked examples 
- Critical thinking prompts: to promote the abilities to evaluate one's own thinking and fill in gaps in knowledge

Incorporating and combining empirically validated learning techniques that match the learning needs of each trainee is fundamental to the overarching approach for the TEMS design concept. Our objective is to bring the best practices of instruction forward by design and to do so by integrating precision assessments of each trainee's engagement via behavioral and physiological assessments.

The strength of the approach comes from a convergence of advanced technologies and best practices from a variety of disciplines. However, that means we will have to tackle many technical, theoretical, and practical challenges to realize a fully implemented system. Among the priorities, is to validate best practices/guidelines that identify which instructional prompts are best suited for a topic, a trainee's scaled competency, and/or learner's style and state. While some guidance exists in for the use of instructional strategies there remains a gap for implementation in a state-based system.

\section{$4 \quad$ Envisioned System}

The TEMS design concept is an innovative instructional design that supports instructors in the management of trainee engagement. Among other objectives, the work described in this paper is attempting to create a field ready system that simultaneously introduces both advanced technology and learning strategies. The TEMS design concept is envisioned as a domain agnostic instructional technology that could be used to support any instructor in achieving their goals, deliver high quality instruction to as many trainees as possible. To support those objectives, we have conceptualized our design concept within a common CBT context that shares many features with a traditional classroom setting, see Figure 3.

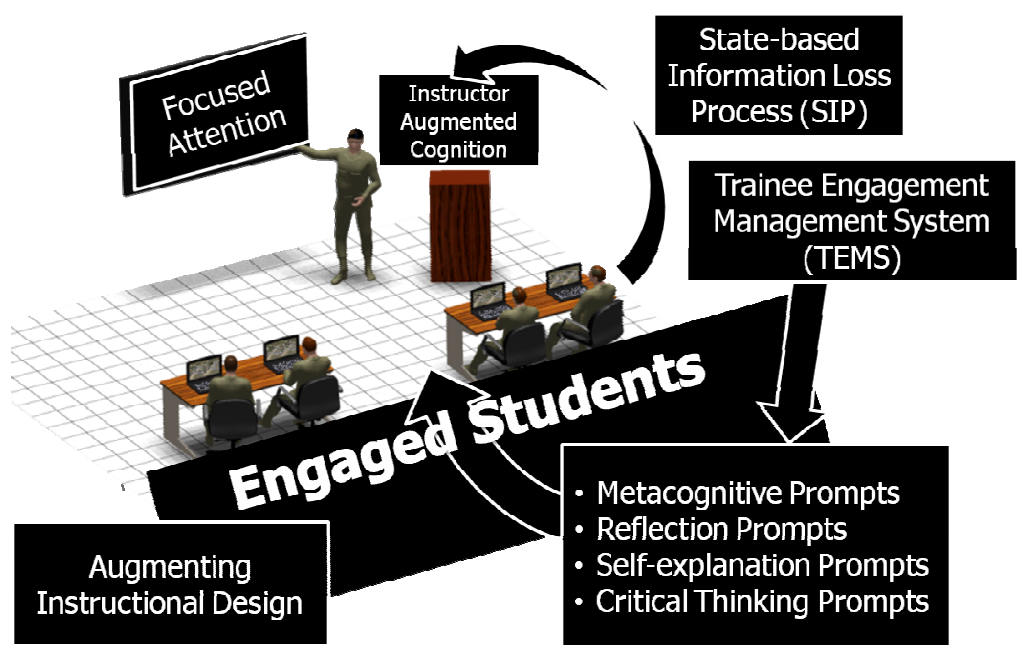

Fig. 3. Traditional Classroom Features 
The basic idea is to provide instructors real-time assessments of engagement for each trainee in the instructional setting. Visualizations of engagement will be displayed on an Instructor Operator Station (IOS) allowing instructors to get a top-level view of trainee engagement at a single location. When a trainee's engagement drops below an acceptable level, the instructor can easily identify which trainee is not properly engaged. When a state-based change is observed the instructor may choose to take steps to re-engage that trainee or they may decide see if the trainee self-corrects to regain engagement. The TEMS design concept embraces a human systems integration philosophy that supports, not replaces, instructors to promote tailored instruction in CBT environments.

\section{Discussion}

A mix of technical and technological change throughout the military contexts imposes a great deal of responsibility on military personnel to reach and maintain mission readiness. Effectively and efficiently imparting mission-critical KSAs is a first order goal of instructors across the military services. Under ideal one-on-one conditions it can be difficult to optimize that match instructional content and a trainee's unique needs. Individual differences and state-based variations converge at each training session to create a signature experience. Thus, delivery of high quality instruction at each training session requires an adaptive capability that is responsive to the dynamic person variables. To date one-on-one paradigms with highly skilled instructors are the best way to consistently obtain that quality of instruction. However, that paradigm is extremely costly and is not sustainable, or reasonably feasible, in military training settings. Typically, trainee settings include one instructor that is responsible for a large number of trainees with a ratio that is closer to $1: 10$, as compared to $1: 1$; thus, a need for innovative instructional designs persists.

A significant amount of work continues to be devoted to the development of Intelligent Tutoring (IT) systems that can be used to support training in unsupervised contexts and replace live instructors. The explicit goal of IT is to achieve the same level of quality as observed in one-on-one instruction. However, this is not likely to be realized in the near-term as a field-ready solution. In large part, much work remains in the fields of Artificial Intelligence (AI) and Natural Language Processing (NLP) before this worthy goal can be validated and transitioned. For that reason, the AI capabilities required to support the TEMS design concept are comparatively crude. The TEMS design concept only provides indications of engagement that can be derived from commercially available data classifiers. The actual intelligence within the TEMS concept resides within the instructor's expert judgment and highly skilled decision making. As the research community demonstrates advances in AI capabilities future implementations will incorporate intelligent tutoring capabilities; however, the envisioned system is intended to always include roles and responsibilities for a live human instructor. 


\section{References}

1. Yerkes, R.M., Dodson, J.D.: The Relation of Strength of Stimulus to Rapidity of Habitformation. Journal of Comparative Neurology and Psychology 18, 459-482 (1908), http: / / psychclassics.yorku.ca/Yerkes/Law/

2. Vygotsky, L.S., Whorf, B.L., Wittgenstein, L., Fromm, E.: Language and Consciousness. In: Pickering, John, Skinner, Martin (eds.) From Sentience to Symbols: Readings on Consciousness, Harvester Wheatsheaf (1990)

3. Hancock, P.A., Warm, J.S.: A Dynamic Model of Stress and Sustained Attention. Human Factors 31(5), 519-537 (1989)

4. Csikszentmihalyi, M.: Flow: The Psychology of Optimal Experience. Harper Perennial, New York (1991)

5. Paas, F., van Gog, T.: Principles for Designing Effective and Efficient Training of Complex Cognitive Skills (2009), doi:10.1518/155723409X448053

6. Craven, P.L., Tremoulet, P.D., Barton, J.H., Tourville, S.J., Dahan-Marks, Y.: Evaluating training with cognitive state sensing technology. In: Schmorrow, D.D., Estabrooke, I.V., Grootjen, M. (eds.) Augmented Cognition, HCII 2009. LNCS, vol. 5638, pp. 585-594. Springer, Heidelberg (2009)

7. Blackhurst, J.L., Gresham, J.S., Stone, M.O.: The Quantified Warrior. Armed Forces Journal (2012), http: / / www . armedforcesjournal . com/2 012/12/12187387 (retrieved)

8. Vogel-Walcutt, J.J., Bowers, C.A., Marino-Carper, T., Nicholson, D.: Increasing Learning Efficiency in Military Learning: Combining Efficiency and Deep Learning Theories. Military Psychology (2010)

9. Brown, A.: Metacognition, Executive Control, Self-Regulation, and Other more Mysterious Mechanisms. In: Reiner, F., Kluwe, R. (eds.) Metacognition, Motivation, and Understanding, pp. 65-116. Erlbaum, Hillsdale (1987)

10. Lackey, S.: Next-Generation Expeditionary Warfare Intelligent Training (NEW-IT) Program Summary Booklet (2011), http://active.ist.ucf.edu/ LinkClick.aspx?fileticket $=\mathrm{K} 0 \% 2 \mathrm{bcwKl} 0 \mathrm{br} Q \% 3 \mathrm{~d} \& \mathrm{tabid}=430$ 